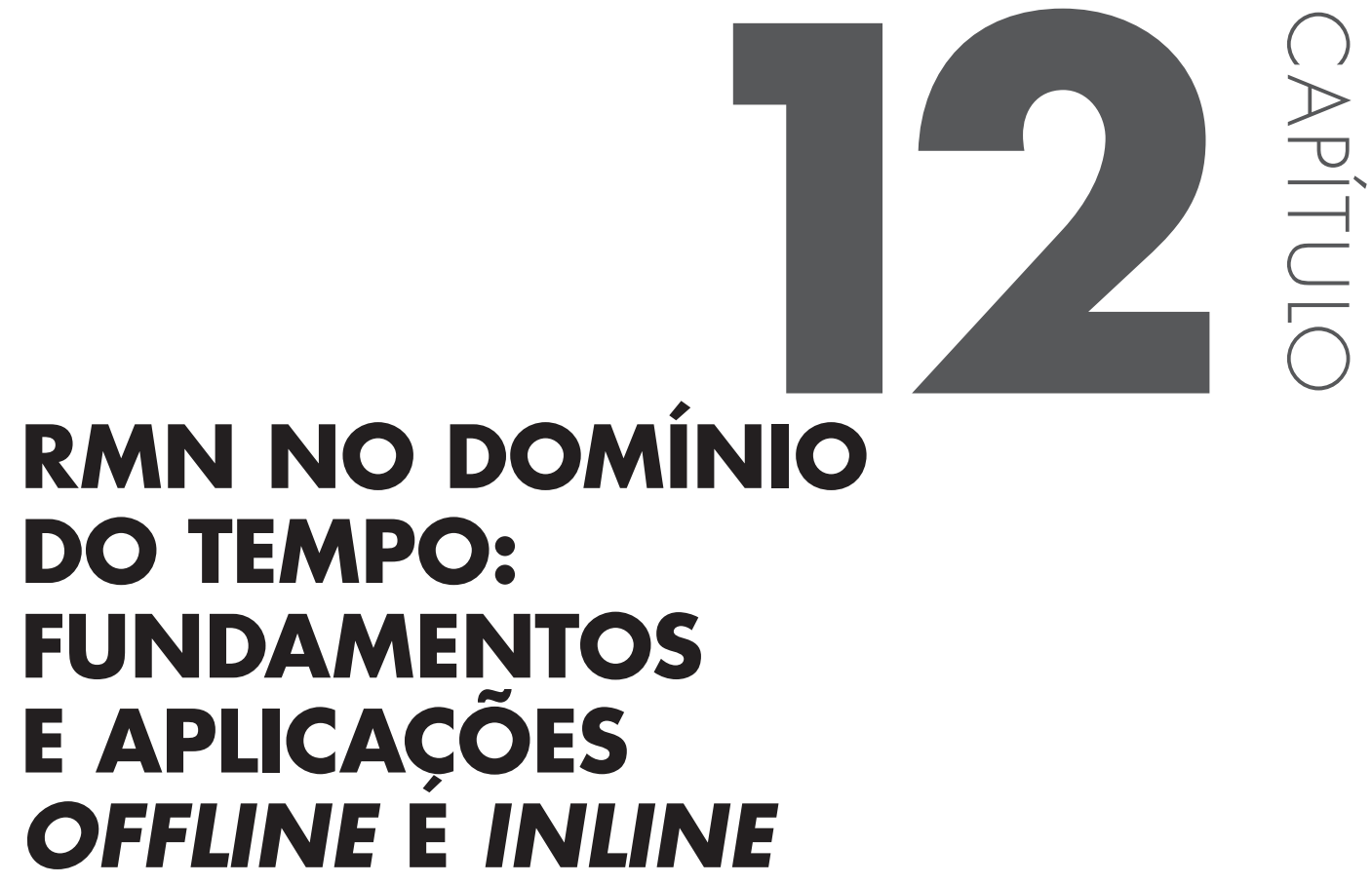

Luiz Alberto Colnago

Fabiana Diuk de Andrade

\title{
12.1 INTRODUC̣ÃO
}

A descrição do fenômeno de ressonância magnética nuclear (RMN) foi publicada em 1946 por dois grupos independentes: o dos físicos Purcell, Torrey e Pound, da Universidade de Harvard, e o de Bloch, Hansen e Packard, da Universidade de Stanford. Logo no início da década de 1950 a RMN já era usada para a solução de problemas químicos. Em menos de dez anos após a sua descoberta, o primeiro espectrômetro de RMN de alta resolução para ${ }^{1} \mathrm{H}$ em onda contínua já estava no mercado ${ }^{1-4}$.

A RMN foi um grande salto para a determinação estrutural de moléculas orgânicas, reduzindo o tempo de elucidação da estrutura de moléculas orgânicas em várias ordens de grandeza ${ }^{5}$. No final dos anos 1960, um novo patamar da RMN foi obtido com a introdução dos espectrômetros pulsados, com a transformada de Fourier (do inglês Fourier transform-nuclear magnetic resonance - FT-NMR) e dos ímãs supercondutores ${ }^{1-3}$. No início da década de 1970, duas novas técnicas foram introduzidas: a RMN multidimensional e a 
tomografia por ressonância magnética nuclear, conhecida atualmente como ressonância magnética.

A RMN multidimensional proporcionou não só um novo salto para a determinação da estrutura de moléculas orgânicas de baixa massa molecular, mas também revolucionou a bioquímica como uma ferramenta de determinação da estrutura tridimensional de proteínas em solução.

A tomografia por ressonância magnética teve seu avanço inicial mais lento que a RMN multidimensional, pois foi necessário o desenvolvimento de toda uma nova tecnologia para a construção dos equipamentos. Diferentemente dos espectrômetros de $\mathrm{RMN}$, os tomógrafos necessitam de ímãs de grande massa e volume, o que consequentemente eleva seus custos. No entanto, no final da década de 1980, experimentos com seres humanos começaram a ser realizados em alguns laboratórios universitários e nas indústrias de equipamentos médicos. Apesar dos altos custos, os tomógrafos de ressonância magnética logo passaram a ser uma das mais importantes ferramentas da medicina, substituindo o tomógrafo de raios $\mathrm{X}$ na maioria dos diagnósticos por imagens.

Devido ao grande impacto que as técnicas de RMN tiveram tanto nas ciências básicas quanto nas aplicadas, vários cientistas foram premiados pela Fundação Nobel por suas descobertas. Em 1952, Felix Bloch e Edward Mills Purcell foram reconhecidos com o prêmio Nobel de Física pela descoberta do fenômeno de RMN; em 1991, Richard Ernst recebeu o prêmio Nobel de Química pelo desenvolvimento da RMN pulsada com transformada de Fourier e RMN multidimensional; em 2002, Kurt Wüthrich recebeu o prêmio Nobel de Química pelo uso da RMN multidimensional para determinar a estrutura tridimensional de proteínas; e, em 2003, Paul Lauterbur e Peter Mansfield receberam o prêmio Nobel de Medicina pela introdução da ressonância magnética por imagem.

Além da química orgânica, bioquímica e medicina, a RMN em alta resolução também é empregada em muitas outras aplicações, em áreas como materiais, química inorgânica, alimentos, análises químicas in vivo, metabolômica, petróleo, entre outras. Uma nova área na qual a RMN começa a se tornar importante é o controle e certificação da qualidade (CCQ) de produtos e processos industriais. Nessa aplicação utilizam-se espectrômetros de RMN de baixo custo, massa e volume, quando comparados com os de RMN em alta resolução em alto campo.

Esses aparelhos são conhecidos como RMN no domínio do tempo (RMN-DT); RMN em baixo campo (RMN-BC) e RMN em baixa resolução (RMN-BR). Essas denominações vêm do fato de os sinais de RMN nesses 
espectrômetros serem analisados no domínio do tempo (DT), ou seja, sem a necessidade da transformada de Fourier. Esses espectrômetros normalmente utilizam ímãs de baixo campo (BC) de até 0,5 T (20 $\mathrm{MHz}$ para o $\left.{ }^{1} \mathrm{H}\right)$, e usam ímãs de baixa homogeneidade, que leva a espectros em baixa resolução (BR), com largura de linhas de até centenas de kHz. Assim, nesses espectrômetros não se observa o efeito do deslocamento químico ou de qualquer outra interação que resolva o espectro em mais de uma linha. Ou seja, independentemente da amostra, só se observa uma linha nesses espectrômetros. Por isso, na RMN-DT se analisa a amplitude do(s) sinal(is) do decaimento livre da indução (do inglês free induction decay - FID) ou a recuperação do seu sinal na forma de eco(s) em função do tempo. Assim, neste capítulo serão descritos os fundamentos e aplicações da RMN-DT desde o modo offline até o inline.

\subsection{FUNDAMENTOS DA RMN}

A RMN é observada quando certos núcleos atômicos, como, por exemplo, ${ }^{1} \mathrm{H},{ }^{13} \mathrm{C},{ }^{19} \mathrm{~F}$, que possuem momento angular ou spin (L) e momento magnético $\mu$, são submetidos a um campo magnético estático, denominado $\mathrm{B}_{0}$, e a um campo magnético oscilante, denominado $\mathrm{B}_{1}$.

O campo magnético $B_{0}$ faz com que $\mu$ precessione" com uma frequência angular denominada frequência de Larmor, $\varpi_{0}$. A frequência de precessão é proporcional ao campo magnético, $\mathrm{B}_{0}$, e à razão magnetogírica, $\gamma$, tal que:

(Equação 12.1) $\quad \boldsymbol{\varpi}_{\mathbf{0}}=\boldsymbol{\gamma} \boldsymbol{B}_{\mathbf{0}}$ ou $\boldsymbol{v}=\boldsymbol{\gamma} \boldsymbol{B}_{\mathbf{0}} / \mathbf{2} \boldsymbol{\pi}$

em que $\gamma$ é uma constante intrínseca de cada núcleo.

$\mathrm{O}$ efeito de $\mathrm{B}_{0}$ sobre $\mu$ também dá origem a uma magnetização resultante, $\mathrm{M}_{0}$, que correspondente ao somatório dos momentos magnéticos no eixo $\mathrm{z}$ (Equação 12.2, Figura 12.1).

\section{(Equação 12.2) $\quad \boldsymbol{M}_{\mathbf{0}}=\sum \boldsymbol{\mu}$}

Para a obtenção do sinal de RMN, o campo magnético oscilante, $\mathrm{B}_{1}$, com frequência $\varpi_{0}$ é aplicado perpendicularmente a $B_{0}$ (Figura 12.1) e na forma de um pulso (espectrômetro pulsado).

* A precessão é um fenômeno físico que consiste na mudança de orientação do eixo de rotação de um objeto. 


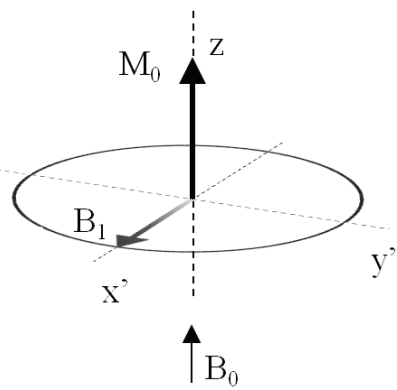

Figura 12.1 Representação do fenômeno de RMN no sistema de coordenadas girantes, no qual o plano xy gira com uma frequência angular próxima a $\varpi_{0}$. Neste sistema, os eixos $x$ e y são denominados $x^{\prime}$ e $y^{\prime}$ para diferenciá-los do sistema de coordenada de laboratório ${ }^{6}$. Na presença de um campo magnético externo $\left(B_{0}\right)$, amostras com núcleos com $L$ e $\mu$ originam uma magnetização resultante, $M_{0}$. 0 campo magnético oscilante, $B_{1}$, aplicado perpendicularmente a $B_{0^{\prime}}$ é usado para excitar a amostra.

O pulso faz com que a magnetização $M_{0}$, que estava originalmente na direção do campo $\mathrm{B}_{0}$ (eixo $\mathrm{z}$ ), gire um ângulo $\theta$, proporcional à duração do pulso $\left(t_{p}\right)$, à intensidade do campo $B_{1}$ e a $\gamma$ (Equação 12.3). O pulso leva a uma magnetização no plano x’y', originando as componentes $M_{x}$ e $M_{y}$.

$$
\text { (Equação 12.3) } \quad \theta=\gamma B_{1} t_{p}
$$

Após o pulso, a magnetização no plano x'y' volta a precessionar em torno de $\mathrm{B}_{0}$ e induz o sinal de $\mathrm{RMN}$ na bobina da sonda, denominado de decaimento livre da indução (FID). Após o pulso, o FID passa por dois processos de relaxação distintos: a relaxação longitudinal $\left(\mathrm{T}_{1}\right)$, que está envolvida no retorno da magnetização ao equilíbrio térmico; e a relaxação transversal $\left(\mathrm{T}_{2}\right)$, que está relacionada ao desaparecimento do sinal no plano x'y'. Esses tempos de relaxação interferem diretamente na quantificação da técnica, largura da linha, decaimento do FID, entre outras variáveis.

\subsubsection{FID}

Como $\mathrm{T}_{1} \geq \mathrm{T}_{2}$, o decaimento do FID em um ímã perfeitamente homogêneo decai com $\mathrm{T}_{2}$. Entretanto, em um aparelho de RMN-DT, o FID de uma amostra líquida ou em solução decai muito mais rapidamente do que o determinado por $\mathrm{T}_{2}$. Nesses aparelhos, o decaimento do FID é governado pelo 
tempo de relaxação efetivo, $\mathrm{T}_{2}{ }^{*}$ (Equação 12.4), que normalmente possui maior contribuição da não homogeneidade do campo magnético $\left(\Delta \mathrm{B}_{0}\right)$ do que do $\mathrm{T}_{2}$ natural da amostra.

$$
\text { (Equação 12.4) } \frac{1}{T_{2}^{*}}=\frac{1}{T_{2}}+\gamma \Delta \mathbf{B}_{0}
$$

A Figura 12.2 apresenta um FID $\left({ }^{1} \mathrm{H}\right)$ de uma amostra heterogênea, contendo componentes no estado sólido (estrutura rígida) e no estado líquido (alta mobilidade). Essas amostras podem ser, por exemplo, sementes oleaginosas, polímeros com monômeros, alimentos, tecidos biológicos, entre outros materiais heterogêneos.

Considerando que a amostra é uma semente oleaginosa, logo após o pulso, o FID correspondente a todos os componentes da semente que contêm hidrogênio, tais como óleo, carboidratos, proteínas e água ${ }^{7}$. $\mathrm{Na}$ expansão de parte da Figura 12.2 é possível observar que a parte inicial do FID (A) decai durante o tempo morto do espectrômetro. O tempo morto é definido pelo período no qual as interferências ocasionadas pelas imperfeições

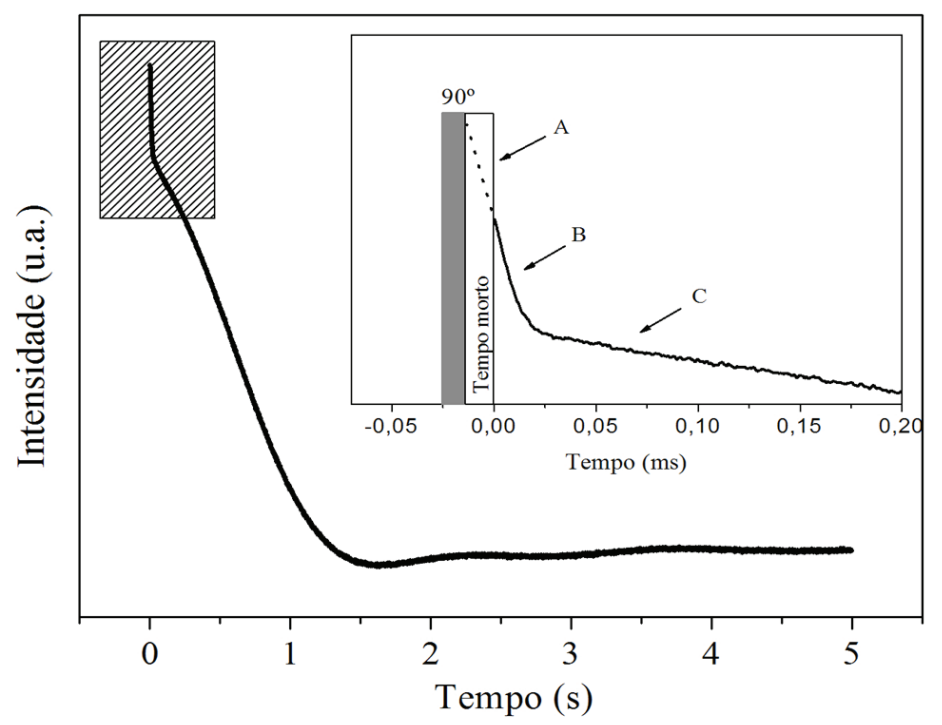

Figura 12.2 FID de uma semente de amendoim após um pulso de $90^{\circ}$ em um espectrômetro de RMN-DT. A expansão do sinal mostra o pulso, 0 tempo morto do espectrômetro e 0 início do sinal FID. A linha tracejada (A) mostra 0 sinal FID durante o tempo morto contendo todos os componentes da semente. 0 FID em (B) mostra parte do sinal dos componentes sólidos sobreposta ao dos componentes líquidos. 0 FID em (C) contém somente 0 sinal dos componentes móveis da semente. 
instrumentais são maiores do que o sinal do FID. Durante o tempo morto de $11 \mathrm{~ms}$, nesse espectrômetro, a maioria do sinal FID dos componentes rígidos da semente, como proteínas, carboidratos e água ligada, decaem a uma fração da intensidade inicial, por apresentarem $T_{2}$ muito curto, da ordem de décimos de microssegundos 7,8 . Consequentemente, apenas uma pequena parte do FID desses componentes (B) pode ser medida. No componente (C) do FID estão apenas os sinais dos componentes no estado líquido, o óleo e água livre. Como eles têm um $\mathrm{T}_{2}$ de dezenas a centenas de milissegundos, o decaimento do FID (C) é governado, principalmente pela não homogeneidade do campo magnético $\left(\Delta \mathrm{B}_{0}\right)$ (Equação 12.4 $)^{7,8}$. A intensidade do FID a algumas dezenas de microssegundos após o pulso tem sido usada para a análise quantitativa de componentes líquidos em amostras homogêneas e heterogêneas. A intensidade do FID ( $\mathrm{RMN}$ de $\left.{ }^{1} \mathrm{H}\right)$ é usada para medir o teor de hidrogênio em combustíveis, teor de óleo, umidade em alimentos, produtos agrícolas, produtos químicos, materiais poliméricos, cimentos, entre outras medidas. O FID do ${ }^{19} \mathrm{~F}$ é usado para determinar o teor de fluoreto em dentifrícios.

\subsubsection{Relaxação longitudinal}

O mecanismo de relaxação longitudinal, spin-rede ou $\mathrm{T}_{1}$, está relacionado ao retorno da magnetização ao estado de equilíbrio térmico, $\mathrm{M}_{0}$. Essa relaxação é resultante da existência de momentos magnéticos transitórios produzidos pelos movimentos rotacionais e translacionais de moléculas da vizinhança, oscilando com frequências apropriadas em torno da frequência de Larmor. A taxa de relaxação $1 / \mathrm{T}_{1}$ é máxima quando a frequência do campo magnético flutuante, provocado pela rotação da molécula $\left(\tau_{c}\right)$, equivale à frequência de Larmor $\left(\tau_{c} \approx 1 / \varpi_{0}\right)$. Fora dessa condição, a relaxação longitudinal tende a ser menos eficiente.

$\mathrm{O}_{1}$ é responsável pela recuperação exponencial do módulo da magnetização, ao longo do eixo z $\left(\mathrm{M}_{\mathrm{z}}\right)$ dado pela Equação 12.5:

$$
\text { (Equação 12.5) } \quad \boldsymbol{M}_{\mathbf{z}}(\boldsymbol{t})=\boldsymbol{M}_{\mathbf{0}}\left[\mathbf{1}-\boldsymbol{e x p}^{\left(-\boldsymbol{t} / \boldsymbol{T}_{\mathbf{1}}\right)}\right]
$$

Assim, para análises quantitativas, o $\mathrm{T}_{1}$ deve ser conhecido ou estimado para evitar a saturação de um ou mais sinais da amostra. Normalmente, é necessário um tempo de espera, $\mathrm{T}_{\mathrm{r}}$, de pelo menos $5 \mathrm{~T}_{1}$ entre cada varredura 
do espectro de RMN para que a magnetização $\mathrm{M}_{\mathrm{z}}$ retorne aproximadamente $99 \%$ de $\mathrm{M}_{0}$.

A Figura 12.3A mostra a sequência de pulsos mais usada para medir $\mathrm{T}_{1}$, a sequência de inversão-recuperação (IR). Essa sequência consiste em um pulso de $180^{\circ}$, para inverter a magnetização, seguido por um tempo $\tau$ (variável) e um pulso de $90^{\circ}$ para medir a intensidade do FID. Essa sequência também é representada esquematicamente por $\left(180^{\circ}-\tau-90^{\circ}\right)_{n}$. A Figura $12.3 \mathrm{~B}$ apresenta um gráfico da variação da intensidade do FID em função de $\tau$.

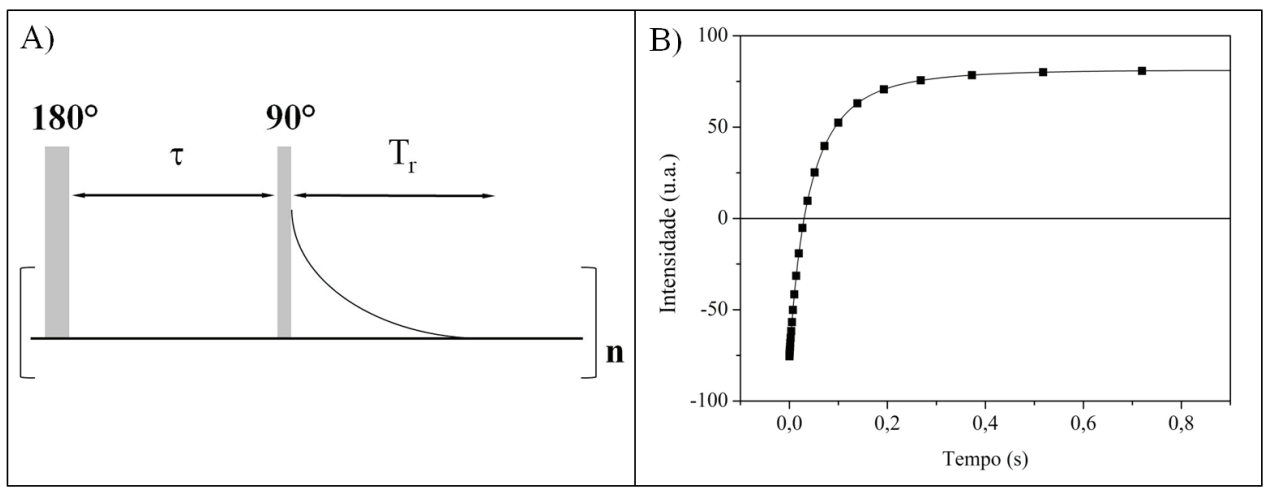

Figura 12.3 (A) Sequência de pulsos inversão-recuperação. (B) Variação da amplitude do sinal em função de $\tau$.

A Figura 12.4 apresenta uma descrição vetorial do comportamento dos spins durante a sequência IR. A Figura 12.4A exibe a magnetização no equilíbrio térmico. $\mathrm{O}$ pulso de $180^{\circ}$ inverte a magnetização da direção z $\left(\mathrm{M}_{\mathrm{z}}\right)$ para $-\mathrm{z}$ (Figura 12.4B). Durante o tempo $\tau$, entre B e C, ocorre a relaxação. Dependendo do tempo $\tau$ e do $\mathrm{T}_{1}$, há uma distribuição da magnetização entre z e $-z$, conforme vetores cinza e preto da Figura 12.4C. Para valores de $\tau<<\mathrm{T}_{1}$, a componente $-\mathrm{z}$ (preta) é bem maior do que a componente $\mathrm{z}$ (cinza). Para valores de $\tau<<\mathrm{T}_{1}$, a componente em z é maior do que a $-\mathrm{z}$. Se $\tau \sim 5 \mathrm{~T}_{1}$, a componente $\mathrm{z}$ atinge aproximadamente $99 \%$ do valor de $\mathrm{M}_{0}$. Para detectar as componentes no eixo $z$, que são dependentes de $\tau$ e de $T_{1}$, aplica-se um pulso de $90^{\circ}$ para rotacionar a magnetização para o plano x'y' (Figura 12.4D), onde se faz a medida da intensidade do sinal de RMN. A componente que estava no eixo z (Figura 12.4C), vai para a direção y (cinza), e a componente que estava na direção $-z$ vai para a direção -y (preta). Como o sinal de RMN é a resultante da magnetização no plano x’y', o sinal de RMN 
na sequência IR varia de negativo a positivo (Figura 12.3B) dependendo do valor de $\tau$ e $T_{1}$. Pode-se calcular o valor de $T_{1}$ com a Equação 12.6.

(Equação 12.6) $\quad I(\tau)=I_{0}\left[1-2 \exp ^{\left(-\tau / T_{1}\right)}\right]$

em que $\mathrm{I}_{0}=$ intensidade do sinal para $\mathrm{T}_{\mathrm{r}}>5 \mathrm{~T}_{1}$.
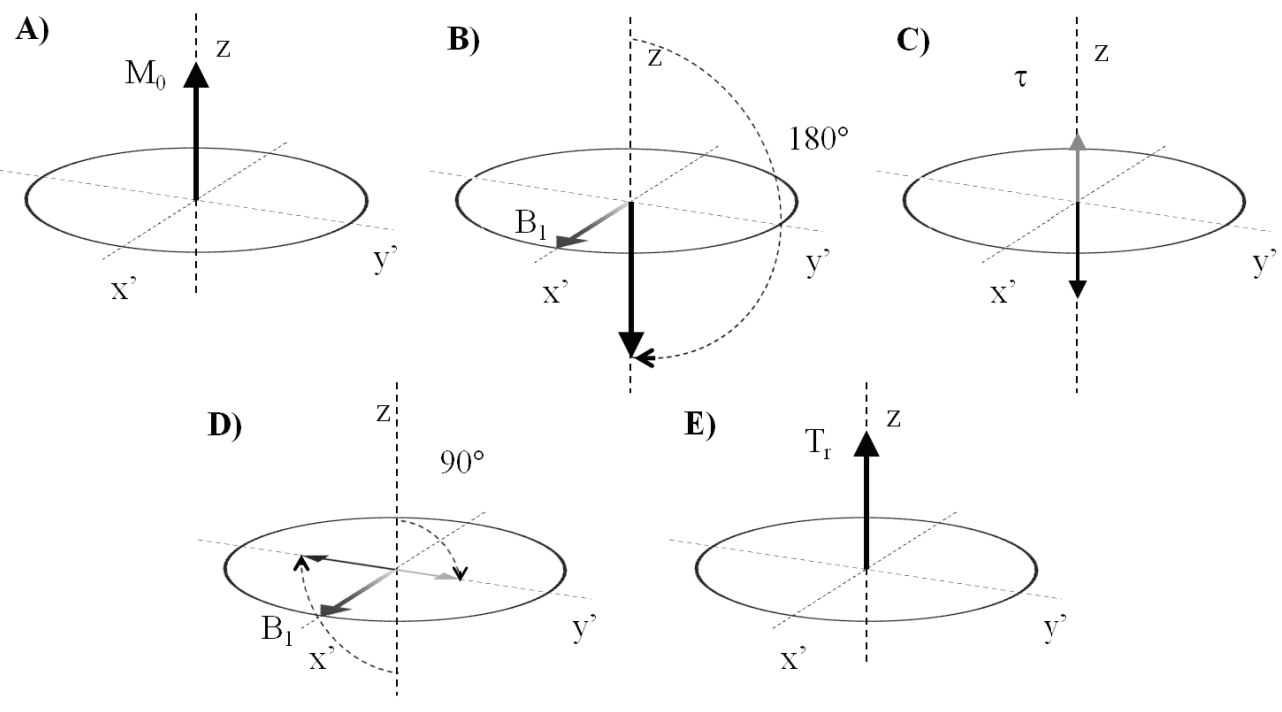

Figura 12.4 Representação vetorial da evolução da magnetização durante os eventos da sequência de pulsos inversão-recuperação.

Medidas de $\mathrm{T}_{1}$ por IR são raramente usadas em análises qualitativa ou quantitativa devido à demora do experimento. No entanto, a sequência IR tem sido usada para suprimir sinais com diferentes valores de $\mathrm{T}_{1}$, ou seja, como um filtro de $\mathrm{T}_{1}$.

\subsubsection{Relaxação transversal}

O tempo de relaxação transversal, spin-spin ou $\mathrm{T}_{2}$, está relacionado à perda de coerência da magnetização no plano x'y'. Essa perda de coerência é atribuída às interações diretas entre os momentos magnéticos individuais dos spins em uma amostra. A relaxação transversal provoca um decaimento exponencial da magnetização transversal, dado pela Equação 12.7: 


\section{(Equação 12.7) $\quad M_{y}(t)=M_{x y} \cos \varpi_{0} t \exp ^{\left(-t / T_{2}\right)}$}

A relaxação transversal, ao contrário da longitudinal, não apresenta um valor máximo de relaxação quando $\tau_{c} \approx 1 / \varpi_{0}$. Ela é maior em moléculas com alta mobilidade e decresce com a redução da mobilidade molecular. Isso justifica porque, na maioria das amostras líquidas ou em solução, $\mathrm{T}_{1} \gg \mathrm{T}_{2}$, enquanto nos sólidos $\mathrm{T}_{1}>\mathrm{T}_{2}$.

Os métodos de medida de $\mathrm{T}_{2}$ são baseados em sequências de eco de spin e serão discutidas em detalhes a seguir.

\subsubsection{Técnicas de eco de spin}

Como demonstrado pela Equação 12.4, a não homogeneidade do campo magnético $\left(\Delta \mathrm{B}_{0}\right)$ de um aparelho de RMN-DT faz com que o FID decaia muito mais rapidamente do que pela dependência de $T_{2}$. Para eliminar a interferência da não homogeneidade na medida do $T_{2}$, Hahn propôs em 1950 uma sequência de dois pulsos de $90^{\circ}$ separados por um tempo $(\tau)$. Essa sequência leva à formação de um sinal de RMN a um tempo $\tau$ do segundo pulso, ou $2 \tau$ do primeiro pulso. Ele denominou esse sinal como eco devido à sua semelhança com o eco acústico. Demonstrou que a amplitude do eco, para vários valores de $\tau$, era independente da não homogeneidade do campo magnético. Desse modo, foi possível determinar o valor de $\mathrm{T}_{2}$ em espectrômetro pulsado, mesmo usando ímãs heterogêneos. Hahn também observou que a intensidade do eco era atenuada quando se usava materiais poucos viscosos, com alto coeficiente de difusão molecular. Isso ocorria em ímãs muito heterogêneos, ou seja, com gradiente de campo magnético muito intenso, ou quando o tempo $\tau$ era longo. A técnica original de Hahn encontrou poucas aplicações, pois logo em 1954 Carr e Purcell substituíram o segundo pulso de $90^{\circ}$ por um pulso de $180^{\circ}$ (Figura $12.5 \mathrm{~A}$ ).

A sequência de pulso de Carr-Purcell, $90^{\circ}-\tau-180^{\circ}$ (Figura 12.5A), denominada método A ou eco de spin, produz um FID após o primeiro pulso, cujo decaimento é governado por $\mathrm{T}_{2}{ }^{*}$. O pulso de $180^{\circ}$, após o tempo $\tau$, refocaliza a magnetização perdida pela não homogeneidade de campo e gera um sinal (eco) a um tempo $\tau$ do segundo pulso. O eco nessa sequência está defasado de $180^{\circ}$ do FID. Normalmente se usa o sinal no modo magnitude, e o decaimento do eco em função de $\mathrm{T}_{2}$ é representado pela linha pontilhada da Figura 12.5A. 


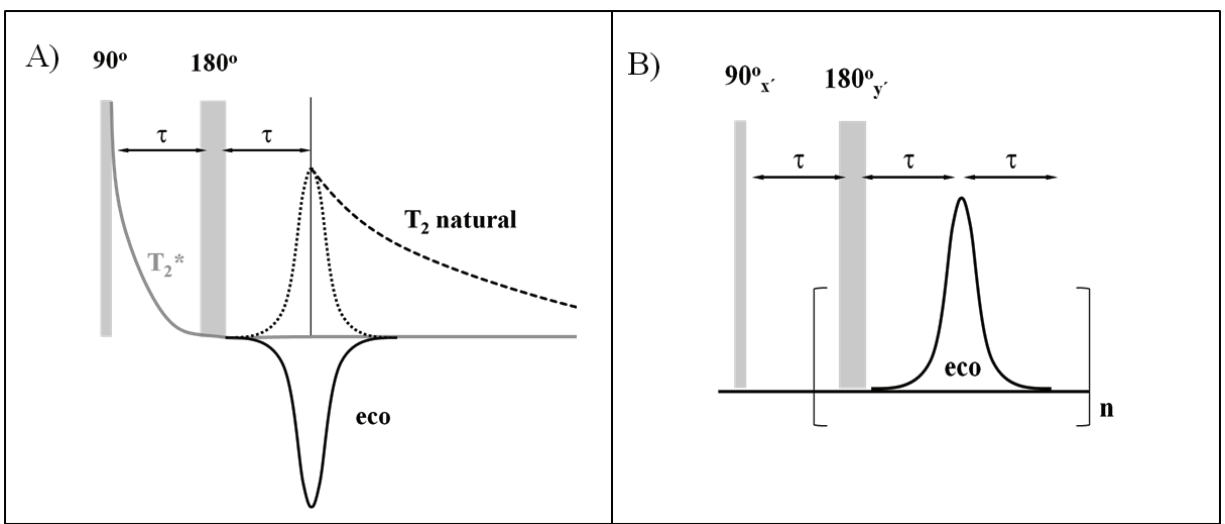

Figura 12.5 (A) Sequência de eco de spin $90^{\circ}-\tau-180^{\circ} .0$ eco com linhas contínuas está no modo fase, com deslocamento de $180^{\circ}$ em relação ao FID, e o eco com linha pontilhada está no modo magnitude. (B) Sequência de pulsos da técnica de CPMG, em que o pulso de $180^{\circ}$ está defasado de $90^{\circ}$ em relação ao primeiro pulso?

Para a medida de $\mathrm{T}_{2}$, tanto no método de Hahn quanto no método A de Carr-Purcell, é necessário fazer a aquisição da intensidade do sinal para vários valores de $\tau$. Porém, entre cada aquisição é necessário esperar um tempo $\left(\mathrm{T}_{\mathrm{r}}\right)$ de pelo menos cinco vezes $\mathrm{T}_{1}\left(\mathrm{~T}_{\mathrm{r}} \geq 5 \mathrm{~T}_{1}\right)$ para o retorno do sistema ao equilíbrio térmico. Isso torna o tempo total de medida de $\mathrm{T}_{2}$ muito longo. Assim como na sequência de Hahn, a intensidade dos sinais de eco na sequência $90^{\circ}-\tau-180^{\circ}$ tem dependência da difusão molecular quando são usadas amostras líquidas com baixa viscosidade, longo tempo $\tau$ e campos magnéticos não homogêneos ${ }^{10}$. Por isso, essa sequência não é indicada para a medida de $\mathrm{T}_{2}$ de componentes em fase líquida em aparelhos de RMN-DT.

No entanto, as técnicas de eco de spin têm sido usadas para análise qualitativa ou quantitativa quando se deseja separar dois componentes baseados na diferença de $\mathrm{T}_{2}$. Esses métodos normalmente medem a amplitude do FID logo após o tempo morto do espectrômetro e a amplitude do eco no tempo $2 \tau$. Esse procedimento é usado para medir a fração sólido-líquido em margarinas e manteigas, umidade e teor de óleo em sementes, entre outras aplicações.

Carr e Purcell também propuseram uma sequência de pulsos denominada Método B ou CP para minimizar ou eliminar o problema de difusão e acelerar a medida de $\mathrm{T}_{2}{ }^{10}$. Nessa sequência, eles não aplicaram apenas um pulso de $180^{\circ}$ após o primeiro pulso de $90^{\circ}$, mas um trem de pulsos de $180^{\circ}$. 
Esse trem de pulsos de $180^{\circ}$, separados por $2 \tau$, gera um eco entre os pulsos de $180^{\circ}$, até o completo desaparecimento do sinal, devido ao $\mathrm{T}_{2}$ natural da amostra. Essa nova sequência permitiu que todo o decaimento do sinal de RMN (ecos) fosse adquirido em uma única sequência de pulsos. Assim, o método CP economiza tempo, pois permite obter os ecos necessários para a medida de $\mathrm{T}_{2}$ em uma única medida, e não é necessário esperar o sistema se relaxar completamente $\left(5 \mathrm{~T}_{1}\right)$ entre cada eco. Além disso, com esse método é possível usar valores de $\tau$ muito pequenos, eliminando consideravelmente o efeito de difusão da amostra na intensidade do eco.

Apesar de toda a vantagem da técnica CP sobre as sequências de eco de spin e eco de Hahn, ela necessita que os pulsos de $180^{\circ}$ sejam muito bem calibrados, para não levar a erros na medida de $\mathrm{T}_{2}$. Para eliminar essa deficiência, Meiboom e Gill (1958) modificaram a sequência CP aplicando os pulsos de $180^{\circ}$ no eixo y', isto é, com uma diferença de fase de $90^{\circ}$ em relação ao pulso de $90^{\circ}$ inicial $^{9}$. Essa nova sequência (Figura 12.5B) aperfeiçoou o método de Carr-Purcell, originando o método chamado $\mathrm{CPMG}^{9}$.

A Figura 12.6 descreve a evolução da magnetização na sequência CPMG. O pulso de $90^{\circ}$, aplicado em eixo x', faz com que a magnetização
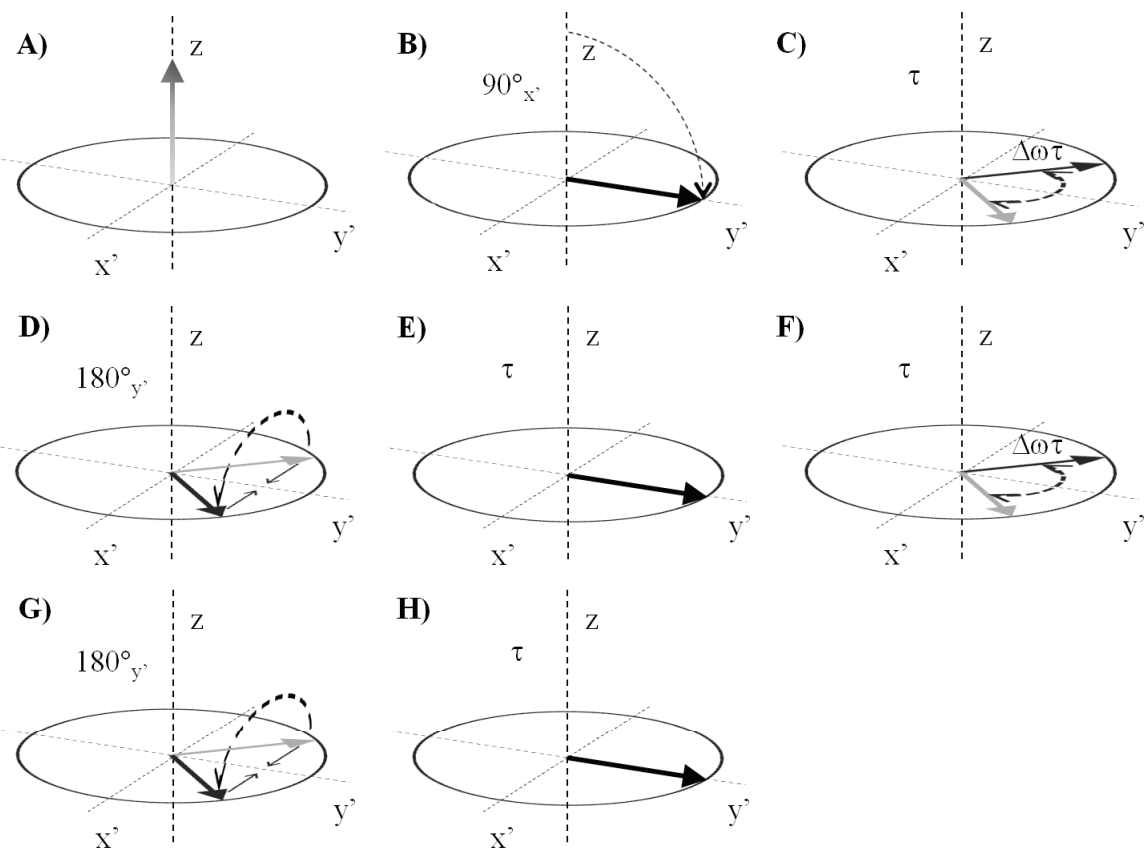

Figura 12.6 Representação vetorial da evolução temporal da magnetização na sequência de pulsos CPMG. 
no equilíbrio térmico (A) gire por esse ângulo, ou seja, até o plano x'y' (B). Durante o tempo $\tau$, as isocromatas de spins (cinza e preta) se defasam devido à não homogeneidade de campo (C). Um pulso de $180^{\circ}$ aplicado em -y' (D) gira as componentes no plano x'y', invertendo a direção dos dois vetores. Como eles mantêm o mesmo sentido de rotação, eles se reencontram (são refocalizados) no eixo $-y$ ', no tempo $2 \tau(E)$, a partir do primeiro pulso. Após isso, ocorre outra defasagem em outro tempo $\tau(\mathrm{F})$. Um segundo pulso de $180^{\circ}$, em $-y^{\prime}$, gira novamente a magnetização $(G)$ e o sinal de eco em $4 \tau(\mathrm{H})^{9}$. Esse procedimento de defasagem e refocalização com pulsos de $180^{\circ}$ ocorre até o desaparecimento completo do sinal em função de $\mathrm{T}_{2}$. A mudança de fase proposta por Meiboom e Gill, além de eliminar o problema de calibração dos pulsos de $180^{\circ}$, também fez com que todos os ecos tivessem uma mesma fase, o que não ocorre com o método CP.

O método CPMG é hoje o método padrão para a medida de $\mathrm{T}_{2}$ dos componentes com alta mobilidade e é largamente usado para análises qualitativas e quantitativas na RMN-DT. É usado na avaliação da qualidade de alimentos, petróleo, produtos agrícolas etc. Essas análises são baseadas, principalmente, nas diferenças entre os tempos de relaxação transversal, $\mathrm{T}_{2}$, dos vários componentes da amostra. Os sinais CPMG vêm sendo analisados através de ajustes mono e multiexponencial, pela transformada inversa de Laplace (ILT) e também por métodos quimiométricos ${ }^{7,8}$.

\subsubsection{Medidas rápidas e simultâneas de $T_{1}$ e $T_{2}$}

Como descrito anteriormente, a medida de $\mathrm{T}_{1}$ em aparelhos de RMN-DT é muito pouco usada devido ao longo tempo necessário para se fazer as medidas. Assim, vários métodos têm sido propostos para reduzir o tempo de medidas, entre os quais estão alguns métodos de precessão livre no estado estacionário (do inglês, steady state free precession - SSFP), que permitem medir tanto $\mathrm{T}_{1}$ quanto $\mathrm{T}_{2}$ em um único experimento. Entre esses métodos, destacamos aqueles denominados CWFP e CP-CWFP.

A sequência continuous wave free precession (CWFP) é composta por um trem de pulsos $90^{\circ}$, separados por um tempo $\tau$, da ordem de $\mathrm{T}_{2}{ }^{*}<<\mathrm{T}_{1}$ e $\mathrm{T}_{2}$. O sinal gerado pela sequência CWFP, a partir do equilíbrio térmico, passa por dois estados transientes antes de atingir o estado estacionário ${ }^{11}$. O primeiro estado transiente é caracterizado por uma alternância de amplitude do sinal entre valores máximos e mínimos e pela constante de tempo denominada $\mathrm{T}_{2}{ }^{*}$. Esse estado é seguido por um estado quasi-estacionário, em que essas 
alternâncias de amplitude desaparecem e o sinal decai exponencialmente até o estado estacionário (EE). A passagem do estado quasi-estacionário para o estacionário CWFP, usando pulsos de $90^{\circ}$, ocorre com uma constante de tempo dada pela Equação 12.8, dependente tanto de $\mathrm{T}_{1}$ quanto de $\mathrm{T}_{2}{ }^{11}$.

$$
\text { (Equação 12.8) } \quad \boldsymbol{T}^{*}=\frac{\mathbf{2} \boldsymbol{T}_{\mathbf{1}} \boldsymbol{T}_{\mathbf{2}}}{\left(\boldsymbol{T}_{\mathbf{1}}+\boldsymbol{T}_{\mathbf{2}}\right)}
$$

A amplitude do sinal no estado estacionário (EE) é constante e não depende de $T_{1}$, mas sim da razão $T_{1} / T_{2}$ de acordo com a Equação 12.912,13:

$$
\text { (Equação 12.9) } \quad \boldsymbol{M}_{\mathrm{EE}}=\frac{\boldsymbol{M}_{\mathbf{0}} \boldsymbol{T}_{\mathbf{2}}}{\left(\boldsymbol{T}_{\mathbf{1}}+\boldsymbol{T}_{2}\right)}
$$

onde $\mathrm{M}_{\mathrm{EE}}$ é a intensidade do sinal no estado estacionário e $\mathrm{M}_{0}$ é a intensidade do sinal no equilíbrio térmico.

Combinando as equações 12.8 e 12.9 , é possível determinar os valores de $\mathrm{T}_{1}$ e $\mathrm{T}_{2}$ em um único experimento ${ }^{11}$, por meio das equações 12.10 e 12.11 , e as amplitudes do sinal após o primeiro pulso (proporcional a $\mathrm{M}_{0}$ ) e no estado estacionário $\left(\mathrm{M}_{\mathrm{EE}}\right)$ e a constante de tempo $\mathrm{T}^{*}$.

(Equação 12.10) $\quad \boldsymbol{T}_{\mathbf{1}}=\frac{\frac{\boldsymbol{T}_{(\alpha=\pi / 2)}^{*}}{2}}{\frac{M_{E E}}{M_{0}}}$

e

(Equação 12.11) $\quad T_{2}=\frac{\frac{T_{(\alpha=\pi / 2)}^{*}}{2}}{1-\frac{M_{E E}}{M_{0}}}$

Além do CWFP, Andrade et al. desenvolveram, em 2012 ${ }^{14}$, um novo método para medir $\mathrm{T}_{1}$ e $\mathrm{T}_{2}$ de maneira rápida e simultânea. Esse método é baseado na sequência Carr-Purcell (CP) com ângulos de refocalização de $90^{\circ}$. Ele foi denominado CP-CWFP devido à sua semelhança com a sequência CWFP, principalmente em relação à sua dependência de $T_{1}$ e $T_{2}$ (14). CP-CWFP é mais eficiente que CWFP para medidas dos tempos de relaxação quanto $\mathrm{T}_{1} \sim \mathrm{T}_{2}$, situação normalmente observada em medidas por RMN em baixo campo.

As sequências CWFP e CP-CWFP têm sido utilizadas para análises quantitativas e qualitativas nas áreas de produtos agropecuários, alimentos in natura e industrializados ${ }^{11,14}$. 


\subsubsection{Análise dos componentes sólidos por RMN-DT}

Até agora, a maioria das análises foram realizadas em amostras líquidas, soluções ou do componente líquido em amostras heterogêneas. A análise de materiais sólidos ou do componente sólido em amostras heterogêneas é bem mais complexa, uma vez que o sinal de RMN decai muito rapidamente.

Como visto anteriormente, a obtenção do FID de componentes sólidos é limitada pelo tempo morto do espectrômetro. Assim, tem se desenvolvido espectrômetros com tempo morto cada vez menores (atualmente, em alguns ms) e de métodos de eco que refocalizem o sinal dos componentes sólidos ${ }^{15}$. Os espectrômetros com tempo morto muito curto ainda não são de uso geral, e, por isso, os métodos de refocalizar o sinal dos componentes rígidos têm sido mais usados ${ }^{15}$.

O primeiro método ou sequência de pulso usada para minimizar o efeito do tempo morto no sinal do componentes sólido foi o eco de sólidos. Essa sequência consiste em um pulso de $90^{\circ}$ no eixo $x^{\prime}\left(90^{\circ}{ }^{\circ}\right)$, um tempo $\tau$ e um pulso de $90^{\circ}$ no eixo y' $\left(90^{\circ}{ }_{\mathrm{y}}\right)$. Essa sequência de pulsos refocaliza um sinal de RMN, na forma de um eco, cuja intensidade no tempo $\tau$, após o segundo pulso, contém o sinal dos componentes sólidos. Mais recentemente, Maus e colaboradores propuseram a sequência chamada magic-sandwich echo com o decaimento do FID (MSE-FID) (Figura 12.7A). Essa sequência, apesar de sua complexidade, com vários tempos de espera e pulsos com diferentes fases, pode ser implementada na maioria dos espectrômetros de RMN-DT comerciais. Sua principal vantagem é recuperar uma maior parte do sinal dos componentes sólidos, em comparação com a sequência eco de sólidos. Com isso, a sequência MSE-FID é, atualmente, a melhor maneira de se obter o sinal tanto dos componentes sólidos quanto dos líquidos de uma amostra heterogênea ${ }^{15}$.

Apesar da simplicidade e popularidade dos experimentos de eco de spin e CPMG para líquidos, existem sérias críticas sobre a análise quantitativa de sólidos no que diz respeito à interpretação das curvas de relaxação em termos de acoplamentos residuais e tempos de correlação dos processos dinâmicos envolvidos ${ }^{10-12}$. Os problemas mais comuns se referem à validade dos modelos usados para ajustar as curvas de relaxação, interdependência dos parâmetros de ajuste e sensibilidade a fatores que influenciam a relaxação, mas não são cobertos pelos modelos. Particularmente, a presença de regiões dinamicamente restritas faz com que a interação dipolar residual $\mathrm{H}-\mathrm{H}$ se torne dominante, não sendo refocalizada nos ecos de CPMG. 
Para minimizar esses problemas, têm sido desenvolvidas sequências de pulsos que fazem uma melhor quantificação dos acoplamentos dipolares residuais. Essas sequências são combinações de ecos de Hahn e ecos sóli$\operatorname{dos}^{16}, \mathrm{RMN}$ de difusão de spins bidimensional ${ }^{17} \mathrm{e}$, mais recentemente, técnicas de ${ }^{1} \mathrm{H}$ de duplo quantum (HDQ) e múltiplo quantum (HMQ) ${ }^{18-20}$.

\subsubsection{RMN de difusão de spins}

Os experimentos de difusão de spins são baseados na transferência de magnetização via interação dipolar homonuclear, que ocorre através de processos de troca dos estados $\alpha \beta$ por $\beta \alpha$ e vice-versa (flip-flop). Os estados $\alpha$ e $\beta$ são os estados de menor e maior energia, respectivamente. O experimento de difusão de spins proposto inicialmente por Goldman e Shen consiste na seleção prévia da magnetização de ${ }^{1} \mathrm{H}$ móvel do material (por exemplo, fase com cadeias de alta mobilidade molecular), seguido do monitoramento da difusão dessa magnetização para os núcleos de ${ }^{1} \mathrm{H}$. A taxa a que tal difusão ocorre está correlacionada com a constante de difusão entre os núcleos de ${ }^{1} \mathrm{H}$ da fase móvel e da fase rígida, que, por sua vez, depende da intensidade do acoplamento dipolar correspondente, do tamanho dos domínios formados por cada fase e da presença de gradiente de mobilidade da fase móvel.

Uma forma mais eficiente de se realizar o experimento de Goldman-Shen é através da utilização de um filtro dipolar, que consiste de um trem de pulsos de $90^{\circ}$, espaçados por um tempo $\tau$, e uma ciclagem de fases tal como mostrado na Figura $12.7 \mathrm{~B}^{21}$. Neste caso, o filtro dipolar promove a seleção da fase móvel, já que durante esse período a magnetização da fase rígida defasa rapidamente no plano transversal. Enquanto que a da fase móvel permanece. Após a seleção pelo filtro dipolar, a magnetização da fase móvel é transferida para o eixo $\mathrm{z}$ durante $\mathrm{t}_{\mathrm{m}}$, onde a difusão de spins pode acontecer. Após $\mathrm{t}_{\mathrm{m}}$ a magnetização é novamente transferida para o plano transversal onde é detectado. Monitorando o sinal da fase móvel como função de $t_{\mathrm{m}}$ é possível obter a taxa de difusão de spins (taxa com que a magnetização da fase móvel diminui devido à difusão para a fase rígida) e a partir daí, a dimensão do domínio formado pela fase móvel. É ainda possível fazer o experimento inverso, ou seja, seleciona-se a magnetização da fase rígida com uma sequência de pulsos adequada e monitora-se a difusão para a fase móvel.

Estudos específicos com a aplicação dessa sequência no estudo de homopolímeros demonstraram que é possível utilizá-la para descrever a dinâmica molecular de polímeros que contenham grupos químicos com significantes 
diferenças de mobilidade ${ }^{22}$ Esse método é utilizado para investigar os tamanhos de domínios (tais como regiões de crosslinks, por exemplo) e morfologia de polímeros heterogêneos ${ }^{23}$.

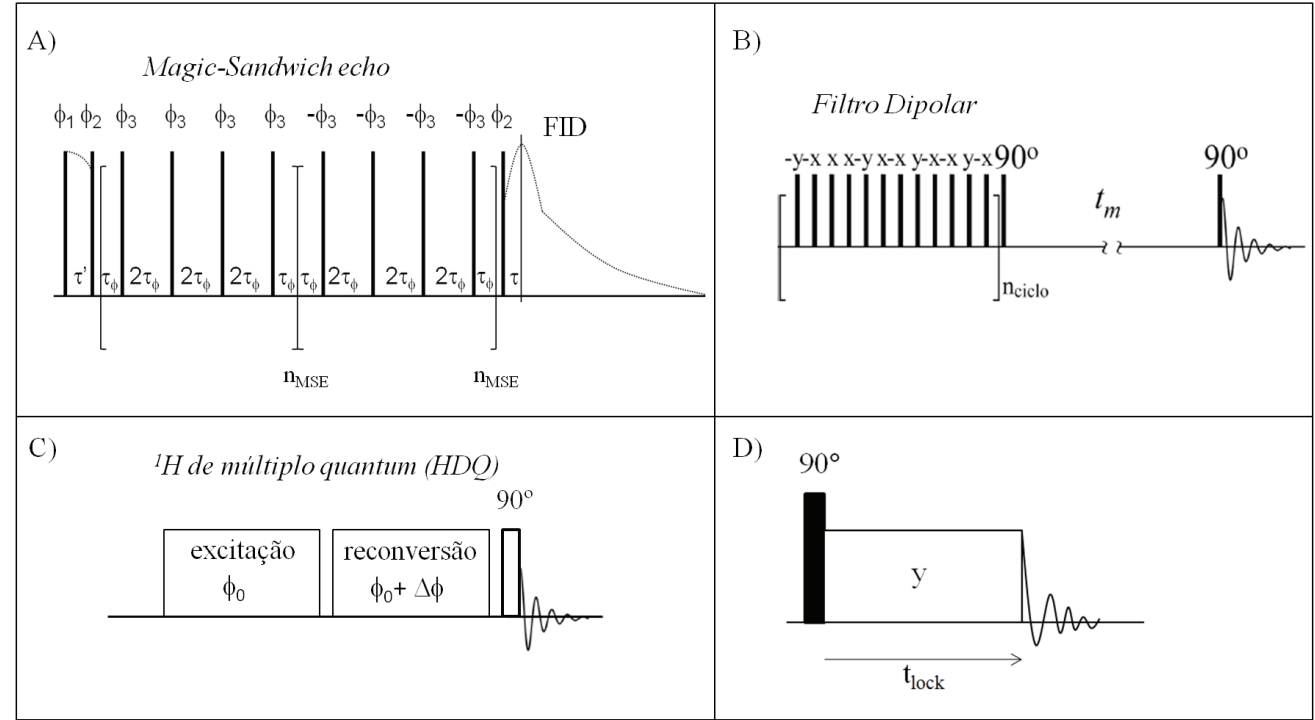

Figura 12.7 (A) Sequência magic-sandwich echo (MSE) combinada com FID. (B) Sequência de difusão de spins ${ }^{21}$. (C) Sequências de pulsos utilizada no experimento de HMQ. (D) Sequência de pulso spin-lock, $\dagger_{\text {lock }}$ corresponde ao tempo de aplicação de $B_{\text {ISL }}$.

\subsubsection{2 'H de duplo quantum (HDQ) e múltiplo quantum (HMQ)}

Nas técnicas de RMN de duplo quantum (HDQ) são aplicadas sequências de pulsos complexas (Figura 12.7C). O sinal resultante dessa sequência de pulsos depende da evolução temporal da magnetização, na presença de coerência de duplo quantum, que só aparecem quando existe acoplamento dipolar residual.

No período de excitação, a sequência de pulsos maximiza a geração de coerências de múltiplo quantum que evoluem durante esse período (Figura 12.7C). Em seguida, no período de reconversão, essas coerências são reconvertidas em coerências de quantum único, gerando uma magnetização que pode ser detectada e cuja amplitude é modulada por um fator que depende da evolução das coerências de múltiplo quantum. A seleção de uma coerência específica pode também ser realizada através de ciclagem de fase. Em 
campo magnético baixo, a quantificação é realizada monitorando a amplitude do sinal no domínio do tempo como função do período de excitação das coerências de múltiplo quantum.

$\mathrm{Na}$ técnica de CPMG, a informação estrutural e dinâmica é quantificada através de um único decaimento $\left(\mathrm{T}_{2}\right)$. Na técnica de HMQ, as curvas em que todas as coerências são excitadas só dependem da dinâmica do sistema, enquanto que, nas curvas onde o sinal obtido pela excitação de, somente uma ordem específica de coerência, é normalizado pelo sinal referente à excitação de todas as coerências e só depende das características estruturais do sistema (densidade de regiões com dinâmica restrita).

Através de modelos específicos é possível obter diretamente as intensidades desses acoplamentos, o que tem correlação direta com a quantidade de hidrogênios dinamicamente restritos na amostra, com parâmetros físicos como a densidade de crosslinks em elastômeros. O mesmo esquema experimental também provê acesso a curvas que dependem da soma de coerências de várias ordens (coerências de múltiplo quantum).

Essa abordagem fornece informações quantitativas sobre a estrutura e heterogeneidades, por exemplo, a mobilidade das cadeias, sendo um marcador sensível do início de gelificação em géis no conteúdo de fração móvel, na estrutura da rede e da mobilidade das cadeias durante o curso e o fim da gelificação. O decaimento dessas curvas depende diretamente de propriedades dinâmicas do sistema e, portanto, elas podem ser usadas para analisar essas propriedades ${ }^{18}$.

Desse modo, utilizando tais técnicas, tanto informações sobre a distribuição de acoplamentos dipolares residuais quanto sobre dinâmica molecular podem ser obtidos com melhor precisão que a oferecida pelas técnicas convencionais. Outra vantagem dos métodos de HMQ é que podem também ser realizados de forma eficiente em campos magnéticos baixos ${ }^{20}$.

\subsubsection{Spin-lock}

O método spin-lock (SL) permite o estudo da relaxação longitudinal no referencial girante $\left(T_{1} \rho\right)$ em campos magnéticos muito baixos sem sacrificar a razão sinal/ruído. A técnica consiste na aplicação inicial de um pulso de $90^{\circ}{ }^{\prime}$, que cria uma magnetização ao longo de y' no referencial de coordenadas girantes. Então um pulso contínuo é aplicado em y', denominado de pulso de spin-lock $\left(\mathrm{B}_{1 \mathrm{SL}}\right)$, durante um tempo, $\mathrm{t}_{\text {lock }}$, que trava os spins no plano x'y', devido ao campo criado por esse pulso (ao longo de y') (Figura 12.7D). 
$\mathrm{B}_{1 S \mathrm{~L}}$ age na magnetização no referencial rotativo do mesmo modo que $\mathrm{B}_{0}$ age na magnetização no equilíbrio térmico. Assim, ele mantém a magnetização ao longo do eixo y' durante o intervalo de tempo em que é aplicado ${ }^{24,25}$.

Durante o pulso de spin-lock não há magnetização ortogonal a $\mathrm{B}_{1 \mathrm{SL}}$, e a magnetização travada no eixo y' relaxa ao longo da direção de $B_{1 S L}$, com uma constante de tempo denominada $\mathrm{T}_{1} \rho$. Uma vez que a relaxação no referencial rotativo é semelhante à relaxação longitudinal em $\mathrm{B}_{0}, \mathrm{~T}_{1} \rho$ apresenta valores muito próximos de $\mathrm{T}_{1}$ medido em um campo magnético muito baixo, na ordem de $B_{1}$. Por outro lado, $T_{1} \rho$ pode apresentar valores próximos de $\mathrm{T}_{2}$. Foi demonstrado que para $\tau$ (tempo entre ecos) muito curtos ou iguais a zero, o trem de pulsos de refocalização de Carr-Purcell (CP) se aproxima da condição de spin-lock, e $\mathrm{T}_{1} \rho$ medido em $\mathrm{B}_{1 \mathrm{SL}}$ muito baixo apresentou valores próximos de $\mathrm{T}_{2}$ medido nessa condição.

A relação entre o tempo de relaxação $T_{1} \rho$ e a distribuição de frequência da mobilidade molecular é similar ao tempo de relaxação $\mathrm{T}_{2}$, onde $\omega_{\text {eff }}$ é a frequência do campo efetivo spin-lock, $\gamma \mathrm{B}_{\text {eff }}$, que para $\mathrm{T}_{1} \rho$ em ressonância é igual a $\gamma \mathrm{B}_{1 \mathrm{SL}}$.

O termo densidade espectral de frequência zero é substituído por $\omega_{\text {eff }}$. De acordo com a Equação 12.12, na presença de mobilidade molecular lenta, $\mathrm{T}_{1} \rho$ é dominado por tempos de correlação $\tau_{\mathrm{c}}$ igual a $1 / 2 \omega_{\text {eff }}$ em vez de $1 / \omega_{0}$ ou $1 / 2 \omega_{0}$ como para $T_{1}{ }^{25}$ Considerando as informações de mobilidade lenta e a correlação com os valores de $\mathrm{T}_{1}$ e $\mathrm{T}_{2}$ em $\mathrm{B}_{0}$ muito baixos, $\mathrm{T}_{1} \rho$ se apresenta como uma alternativa para análises de estruturas rígidas.

(Equação 12.12) $\frac{1}{T_{1 \rho}}=K\left[\frac{3}{2} \frac{\tau_{C}}{1+4 \omega_{e f f}^{2} \tau_{C}^{2}}+\frac{5}{2} \frac{\tau_{C}}{1+\omega_{0}^{2} \tau_{C}^{2}}+\frac{\tau_{C}}{1+4 \omega_{0}^{2} \tau_{C}^{2}}\right]$

\subsubsection{Experimentos bidimensionais no domínio do tempo}

Assim como na RMN em alta resolução, também é possível realizar experimentos bidimensionais com a RMN-DT. A grande diferença entre os experimentos bidimensionais em alta resolução e no domínio do tempo é como os espectros são processados. Nos experimentos em alta resolução, normalmente se aplica a transformada de Fourier em duas dimensões. Já no domínio do tempo, o processamento dos sinais é realizado com a transformada inversa de Laplace em duas dimensões.

Atualmente, o número de experimentos bidimensionais da RMN-DT é de menos de uma dezena. Os experimentos mais usados são os que 
correlacionam $\mathrm{T}_{1}$ versus $\mathrm{T}_{2}$ e $\mathrm{T}_{2}$ versus difusão. Para maiores informações sobre esses experimentos e aplicações, ver Hürlimann et al. $(2006)^{26}$ e Song et al. $(2002)^{27}$.

\subsection{APLICAC̣ÃO DA RMN DESDE A ANÁLISE OFFLINE ATÉ A INLINE}

As análises físico-químicas utilizadas no setor industrial são classificadas em offline, atline, online e inline, dependendo de como e onde são feitas. As definições para esses termos podem segundo o autor. Neste capítulo, utilizaremos as definições propostas por Bonastre e colaboradores. ${ }^{28}$.

As análises offline e atline são caracterizadas pela necessidade de se remover a amostra da linha de produção e transportá-la manualmente até o instrumento de medida.

Nas análises offline, a amostra é analisada em uma instalação centralizada, com instrumentação sofisticada e geralmente automatizada. As vantagens dessa abordagem incluem a economia e a eficiência no tempo de uso dos instrumentos. A principal desvantagem é o longo tempo entre a coleta da amostra e o resultado da análise.

$\mathrm{Na}$ análise atline, o analisador é instalado próximo da linha de produção. As vantagens da análise atline, se comparada com a offline, são a rapidez da análise (quase em tempo real), o emprego de um instrumento mais simples e de menor custo, facilidade de manutenção e operação. No entanto, a instrumentação necessita ser mais robusta e normalmente se faz o monitoramento de poucos parâmetros do processo.

$\mathrm{Na}$ análise online, utiliza-se um sistema automatizado para coleta e transporte da amostra, do processo até um instrumento analítico. O processo online pode ser subdividido em duas categorias: o modo intermitente, ou "stop and flow", em que a amostra é analisada de tempos em tempos, e o método contínuo, no qual se faz um "by-pass" para coleta da amostra, que flui continuamente através do instrumento.

Embora as análises online tenham vantagens sobre os processos offline e atline, elas requerem uma interface confiável e podem necessitar de condicionamento da amostra. A maior desvantagem das análises online é a necessidade de construir uma linha analítica separada, que direcione as amostras para o instrumento a uma temperatura e pressão adequadas.

Já a análise inline é feita in situ, ou seja, diretamente na linha de produção ou reator, sem necessidade de coletar amostra. Normalmente, o analisador 
ou sensor fica inserido no processo (reator), o monitoramento é contínuo e usam-se sensores simples e de baixo custo.

A Tabela 12.1 apresenta um resumo das vantagens e desvantagens da aplicação dos tipos de análise físico-químicas no setor industrial.

Tabela 12.1 Vantagens e desvantagens dos tipos de análises físico-químicas utilizadas no setor industrial

\begin{tabular}{ccc} 
& \multicolumn{1}{c}{ VANTAGENS } & DESVANTAGENS \\
\hline Offline & $\begin{array}{c}\text { Instalação central, instrumentação sofisticada, } \\
\text { análise de muitos parâmetros }\end{array}$ & $\begin{array}{c}\text { Coleta e transporte de amostra até analisador } \\
\text { manualmente, longo tempo para resultado }\end{array}$ \\
\hline Atline & $\begin{array}{c}\text { Menor custo, instrumentação simples, } \\
\text { mais rápida que offline }\end{array}$ & $\begin{array}{c}\text { Coleta e transporte de amostra até } \\
\text { analisador manualmente, monitoramento } \\
\text { de menos parâmetros que offline }\end{array}$ \\
\hline Online & $\begin{array}{c}\text { Sistema automático de coleta de amostra, } \\
\text { resultado quase instantâneo }\end{array}$ & $\begin{array}{c}\text { Linha analítica separada do processo, monitoramento } \\
\text { de menos parâmetros que offline e atline }\end{array}$ \\
\hline Inline & $\begin{array}{c}\text { Resultado instantâneo, análise do } \\
\text { produto no processo (in situ) }\end{array}$ & Monitoramento de menos parâmetros que offline e atline
\end{tabular}

Essas são definições generalizadas de categorias de análise em controle de processos industriais. Em RMN, a maioria dos autores classifica as aplicações em fluxo, como online, quando a amostra é transportada por meio de uma esteira (materiais sólidos, heterogêneos ou líquidos em embalagens etc.) e em tubulações, principalmente para líquidos.

A maioria das aplicações da RMN no setor industrial são offline ou atline. No modo offline são usados tanto aparelhos de RMN em alto campo ou alta resolução, quanto em baixo campo ou baixa resolução. No entanto, no modo atline, as análises são quase que exclusivamente realizadas em aparelhos de RMN em baixo campo, tanto no domínio do tempo quanto no domínio da frequência.

\subsubsection{Análises em fluxo (online e inline) por RMN}

Fluxo é o escoamento de substâncias que se encontram em qualquer estado (sólido, líquido ou gasoso) e pode ser laminar ou turbulento, compressível ou incompressível, contínuo ou intermitente (stop and flow ${ }^{29}$. Existe também o fluxo denominado plug flow (fluxo em pistão), em que a amostra se move como um bloco, como, por exemplo, uma amostra sólida 
transportada por uma esteira. No caso dos líquidos, isso ocorre quando a amostra é transportada homogeneamente com a ajuda de um pistão ${ }^{7}$.

A primeira análise em fluxo por RMN foi feita por Suryan em $1951^{30}$, poucos anos após a descoberta da RMN em 1946. A amostra líquida fluía pela sonda de um espectrômetro de RMN de $25 \mathrm{MHz}\left({ }^{1} \mathrm{H}\right)$, em onda contínua, sob a força da gravidade. Ele observou um aumento do sinal em até 20 vezes, quando comparado com amostras estáticas. Esse comportamento foi relacionado a vários fatores: relaxação longitudinal $\left(\mathrm{T}_{1}\right)$, velocidade do fluxo e período no interior do campo magnético e da bobina de $\mathrm{RF}^{30}$.

Singer demonstrou em 1959 a aplicação da RMN pulsada in vivo no estudo da velocidade do fluxo sanguíneo na cauda de ratos ${ }^{31}$. Um dos procedimentos foi comparar os tempos de relaxação $\mathrm{T}_{1}$ dos prótons da água no sangue antes e após a interrupção do fluxo sanguíneo com um torniquete. Uma vez que o fluxo resulta em menos saturação, a curva de absorção com fluxo de sangue apresentou maior amplitude. O trabalho de Singer foi precursor da aplicação da RMN em fluxo sanguíneo na medicina ${ }^{31}$.

Em 1962, Ladner e Stacey utilizaram a RMN em fluxo contínuo para analisar a umidade de carvão com um aparelho de onda contínua de 16 $\mathrm{MHz}$ para ${ }^{1} \mathrm{H}^{32}$. O fluxo, sob a força da gravidade, foi controlado a $100 \mathrm{~g}$ por hora, possibilitando a distinção entre os sinais derivados dos hidrogênios do material sólido (carvão) e dos hidrogênios presentes na água associada a esse material. Essa pode ser considerada a primeira aplicação da RMN em fluxo no controle de processo industriais.

Após essas aplicações iniciais, outros trabalhos foram aperfeiçoando a instrumentação e as metodologias utilizadas para medidas em fluxo. Um desses exemplos pode ser visto no trabalho de McIvor (1969), que usou um aparelho comercial de RMN em onda contínua com campo magnético $\mathrm{B}_{0}$ de $1,4 \mathrm{~T}^{33}$. Análises em fluxo em média resolução (largura de linha de $2 \mathrm{~Hz}$ a $3 \mathrm{~Hz}$ ) permitiram a quantificação dos componentes de uma mistura de solventes com pequenas diferenças de deslocamento químico. Para fazer as medidas em fluxo, foi incorporada uma serpentina, na estrutura da sonda, a fim de aumentar o tempo de permanência da amostra no ímã antes da análise. Esse processo é conhecido como pré-magnetização e é necessário para que a amostra fique polarizada antes de entrar na bobina de $\mathrm{RF}^{33}$. Essa configuração promoveu um aumento na razão sinal/ruído e maior precisão nas análises quantitativas.

Grover e Singer desenvolveram em 1971 as primeiras aplicações de RMN em fluxo em medicina ${ }^{34}$. Eles realizaram medidas da velocidade do fluxo sanguíneo no interior do dedo humano, com a sequência de pulsos eco de 
spin. Com a amplitude do eco em função do tempo de eco foi possível determinar a distribuição da velocidade do fluxo sob as condições utilizadas no experimento ${ }^{34}$.

Em 1978, Watanabe e Niki desenvolveram o primeiro trabalho acoplando RMN em alta resolução em fluxo com a técnica de HPLC ${ }^{35}$. Utilizaram um espectrômetro pulsado de $60 \mathrm{MHz}$ para o ${ }^{1} \mathrm{H}$ e o método stop and flow.

Atualmente, sistemas hifenados de RMN-HPLC comerciais estão disponíveis em laboratórios de pesquisa e controle de qualidade, e permitiram análises com os eluentes cromatográficos em fluxo contínuo ${ }^{36}$.

Pearson et al. (1987) descreveram um método online para análise de umidade em trigo ${ }^{37}$. O método é baseado na sequência CPMG a fim de minimizar os efeitos da não homogeneidade do campo magnético, é rápido e insensível à densidade de empacotamento da amostra. No mesmo ano, Renou et al. fizeram determinações rápidas e simultâneas de etanol e açúcar em uma fermentação alcoólica ${ }^{38}$.

Nicholls e De Los Santos desenvolveram em 1991 um sensor de umidade para monitorar o processo de secagem na indústria de moagem úmida de milho utilizando medidas de $\mathrm{T}_{1}$ e $\mathrm{T}_{2}{ }^{39}$. Na configuração do sistema, um sensor de $11 \mathrm{MHz}{ }^{1} \mathrm{H}$ foi suspenso sob uma cavidade em uma calha de transporte. O sensor era preenchido com a amostra deslocando um pistão, que, após a medida, a conduzia novamente à linha de processo ${ }^{39}$.

Chen et al., em 1993, estabeleceram um protocolo para a avaliação da maturidade de abacates utilizando um sensor de RMN online de $85 \mathrm{MHz}$ para o ${ }^{1} \mathrm{H}^{40}$. A razão dos sinais do óleo e da água dos espectros de $\mathrm{RMN}$ unidimensionais e os tempos de relaxação $T_{1}$ e $T_{2}$ da água na fruta foram correlacionados com sua maturidade.

Em 1997 Zion et al. desenvolveram um sensor de RMN online para a identificação de azeitonas com e sem caroços. Eles acoplaram uma esteira que se movimentava a até $25 \mathrm{~cm} \mathrm{~s}^{-1}$, em um espectrômetro de RMN, com ímã supercondutor, com bore de $30 \mathrm{~cm}$ de diâmetro ${ }^{41}$.

Hills e Wrigth (2006) demonstraram que a translação da amostra pode ser utilizada para adquirir os sinais de RMN, sem a necessidade do pulso de excitação ou gradiente de campo ${ }^{42}$. O protótipo foi baseado na inserção de um campo de RF contínuo de $\mathrm{B}_{1}$ transversal ao campo magnético estático $\mathrm{B}_{0}$. O movimento da amostra foi utilizado para excitar e gerar o sinal de RMN.

Nos últimos anos, têm aumentado os esforços empregados no desenvolvimento da RMN onlinelinline de baixo custo para análises em fluxo contínuo em esteiras industriais. Muitos autores relataram que diversos fatores de qualidade em vários tipos de frutas e vegetais podem ser analisados com 
RMN online, tais como amadurecimento, injúrias, escurecimento, infecções, quantidade de açúcar, entre outros ${ }^{43-45}$.

\section{2.3.2 Desenvolvimento da RMN em fluxo no Brasil}

No Brasil, os primeiros trabalhos sobre RMN-DT em fluxo ocorreram no final da década de 1990, quando foi observado que o sinal de RMN utilizava a sequência CWFP ${ }^{12,13}$. Nesse trabalho, foram realizados experimentos com o escoamento laminar de água em um espectrômetro de RMN de 7,5 $\mathrm{MHz}{ }^{1} \mathrm{H}$. O sinal CWFP é muito sensível ao fluxo. A amplitude e fase de um sinal CWFP para uma amostra em fluxo dependem da velocidade e sentido do fluxo, do ângulo de flip e precessão e dos tempos de relaxação. Essa dependência foi demonstrada experimentalmente e teoricamente usando as equações de Bloch $^{13}$.

Colnago et al. (2007) utilizaram a técnica de CWFP para medidas quantitativas do teor de óleo de amostras de semente em fluxo ${ }^{46}$. Nesse experimento, as amostras passaram continuamente em uma esteira a uma velocidade de $13 \mathrm{~cm} \mathrm{~s}^{-1}$, o que permitiu a análise de cerca de cem amostras em 14 segundos. Essa técnica tem potencial para a determinação do teor de óleo em mais de 20 mil sementes intactas por hora e está sendo utilizada na avaliação do teor de óleo em sementes e na seleção de material genético para a produção de biodiesel ${ }^{46}$.

Corrêa et al., em 2009, utilizaram a dependência dos tempos de relaxação $\mathrm{T}_{1}$ e $\mathrm{T}_{2}$ do sinal CWFP para determinar o teor de gordura intramuscular de carne bovina no modo stop and flow ${ }^{47}$. Nesse trabalho, demonstraram que o sinal obtido com a sequência CWFP possui maior correlação $(0,9)$ com o teor de gordura do que o obtido com a sequência CPMG $(0,25)$, que depende somente de $\mathrm{T}_{2}$.

Apesar de a técnica CPMG não possuir uma alta correlação com o teor de gordura intramuscular em carne bovina, ela tem alta correlação com a qualidade do óleo em sementes. Em 2007, Prestes et al. utilizaram a sequência CPMG para o desenvolvimento de um sistema automático e rápido de RMN em baixa resolução para análises qualitativas ${ }^{48}$. Nesse trabalho, a técnica CPMG foi aplicada com a finalidade de medir a qualidade de óleo em sementes intactas para uso na produção de biodiesel. O decaimento exponencial $\mathrm{T}_{2}$ medido com a sequência $\mathrm{CPMG}$ é inversamente proporcional à viscosidade do óleo nas sementes, que, por sua vez, está diretamente relacionado aos teores e tipos de ácidos graxos, entre outras propriedades ${ }^{48}$. 
Como as propriedades do biodiesel, tais como viscosidade, número de cetano e índice de iodo, são dependentes do tipo e da concentração dos ácidos graxos presentes no óleo, foi desenvolvida a aplicação da técnica CPMG para a medida desses parâmetros diretamente nas sementes. As curvas de calibração para a viscosidade e número de cetano apresentaram coeficiente de correlação de 0,94 e 0,92, respectivamente, calculados pela técnica CPMG diretamente em sementes como soja, pinhão-manso, amendoim, girassol, entre outras ${ }^{48}$.

A Figura 12.8 apresenta os decaimentos CPMG obtidos para amostras de semente oleaginosas no modo stop and flow. Para esse tipo de medida a semente deve ficar imóvel, somente durante a análise (stop and flow), sendo possível, ainda assim, medir a qualidade do óleo em mais de mil sementes por hora.

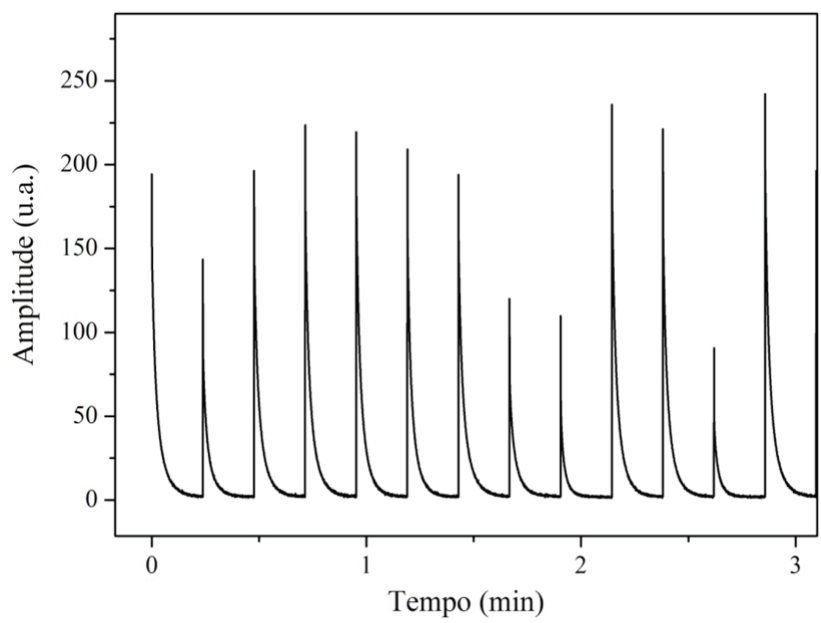

Figura 12.8 Sinal de RMN online obtido pela técnica CPMG de amostras de sementes oleaginosas.

Apesar do grande potencial das técnicas CPMG, tem-se encontrado alguns problemas quando aplicadas online e por longo período, devido a uma sobrecarga no equipamento, principalmente no amplificador de potência e na sonda detectora.

$\mathrm{Na}$ busca de solução para esse problema, foram desenvolvidas técnicas que utilizam, com pulsos de refocalização com ângulos menores que $180^{\circ}$, $\mathrm{CPMG}_{90}$ e CP-CWFP. Essa prática possibilita uma economia de até $75 \%$ de 
potência de RF, com redução dos problemas de superaquecimento em análises em fluxo por $\mathrm{RMN}^{49}$.

\subsection{PERSPECTIVAS}

A utilização da RMN, desde os modos offline até inline, na indústria ainda encontra muitos desafios a serem superados, principalmente no que diz respeito aos problemas de implementação e custos de equipamentos. Por isso, é importante que o desenvolvimento científico e tecnológico prossiga, para tornar cada vez mais economicamente viável o uso da RMN em processos industriais. Portanto, métodos de análise por RMN, rápidos, automáticos, robustos e não destrutivos, deverão ter um grande impacto no controle de processos industriais num futuro próximo.

\subsection{PROTOCOLO PARA DETERMINAC̣ÃO DA QUALIDADE DE ÓLEO EM SEMENTES INTACTAS PÓR RMN}

Materiais

- Aproximadamente 15 sementes oleaginosas com diferentes teores de ácidos graxos, tais como soja, linhaça, milho, amendoim, castanha-do-Brasil, girassol, macadâmia, caju, avelã, abóbora, melancia, rabanete, maracujá, niger, gergelim, noz, pecã, entre outras. Essas sementes podem ser adquiridas em lojas de alimentos e agropecuária.

\section{Equipamentos}

- Cromatógrafo gasoso (CG), com detector de ionização de chama e coluna capilar para separação de ésteres metílicos de ácidos graxos (do inglês fatty acid methyl ester - FAME).

- Espectrômetro de RMN de baixo campo, com porta amostra de pelo menos $10 \mathrm{~mm}$ de diâmetro.

\section{Métodos}

1) Extração do óleo: as sementes oleaginosas desidratadas (5 g a 10 g) devem ser trituradas até a obtenção de um pó bem fino. Então, colocar o pó das 
sementes em um frasco tipo Erlenmyer com aproximadamente $20 \mathrm{~mL}$ de hexano. $\mathrm{O}$ frasco lacrado deve ser agitado por 1 hora em uma capela. Em seguida, o conteúdo do frasco deverá ser filtrado e o solvente com o óleo colocado em um béquer aberto para evaporar (capela). O resíduo deve ser levado a uma estufa a $60{ }^{\circ} \mathrm{C}$, até obtenção de peso constante.

2) Preparação dos FAMES: em um tubo de ensaio, colocar $35 \mathrm{mg}$ do óleo a ser transesterificado, $0,5 \mathrm{~mL}$ de uma solução contendo $0,5 \mathrm{~mol} / \mathrm{L}$ de hidróxido de sódio em metanol e aquecer por 10 minutos a $90{ }^{\circ} \mathrm{C} \mathrm{em}$ banho-maria. Após resfriar a mistura reacional, adicionar $1,5 \mathrm{~mL}$ de uma mistura esterificante preparada com $2 \mathrm{~g}$ de cloreto de amônia, $60 \mathrm{~mL}$ de metanol e $3 \mathrm{~mL}$ de acido sulfúrico concentrado, previamente mantida em refluxo, sob agitação por 15 minutos. Colocar a mistura reacional em banho-maria por 10 minutos, resfriar em banho de gelo e adicione 5,0 mL de n-heptano e $10 \mathrm{~mL}$ de água destilada. Agitar o tubo por alguns minutos e aguardar a ocorrência da separação de fases. Retirar a fase orgânica (superior) que contém os FAMES.

3) Análise dos FAME por GC: usar um volume de injeção de cerca de 1 $\mu \mathrm{L}$ da solução em n-heptano preparado na reação de transesterificação, hidrogênio como gás de arraste, com velocidade linear média de cerca de $31 \mathrm{~cm} / \mathrm{s}$ e nitrogênio como gás auxiliar com aproximadamente $2 \mathrm{~mL} /$ min. Usar a temperatura do injetor e detector de $250{ }^{\circ} \mathrm{C}$, temperatura inicial do forno de $60^{\circ} \mathrm{C}$ por 8 minutos, com taxa de aquecimento de 8 ${ }^{\circ} \mathrm{C} / \mathrm{min}$ até $220{ }^{\circ} \mathrm{C}$, mantendo a esta temperatura até o final da análise. Utilize uma coluna capilar de sílica fundida LM-100 (Carbowax 20M, polietileno glicol) ou equivalente, com $30 \mathrm{~m}$ de comprimento. A quantificação dos FAME é realizada pela normalização (100\%) da área dos picos cromatográficos.

4) Cálculo do índice de iodo, número de cetano e viscosidade do óleo a partir do teor de FAMES (CG): o índice de iodo (II), número de cetano $(\mathrm{CN})$ e viscosidade $(\mu)$ são calculados pelas equações de 12.13 a 12.15 , respectivamente.

(Equação 12.13) $I V=\sum \frac{254 \times D \times A_{i}}{M W_{i}}$

(Equação 12.14) $\ln \mu_{0}=\sum_{i=1}^{n} y_{i} \ln \mu_{i}$

(Equação 12.15) $\quad C N=\frac{46,3+5458}{S N-0,225 \times I V}$ 
em que $D$ é o número de ligações duplas, Ai é a porcentagem de cada FAME determinado por CG, MW $i$ é a massa molecular de cada FAME, $\mu i$ e $\mu_{0}$ a viscosidade cinemática a $40{ }^{\circ} \mathrm{C}$ de cada FAME e da mistura de FAMES, respectivamente; $\mathrm{y}_{\mathrm{i}}$ é $\mathrm{A}_{\mathrm{i}} / 100$ e SN é $\Sigma\left(560 \mathrm{x} \mathrm{A}_{\mathrm{i}}\right) /$ MW. Os valores da viscosidade podem ser obtidos em Prestes et al. $(2007)^{48}$.

5) Análise das sementes por RMN: após calibrar os pulso de $90^{\circ}$, usar uma sequência CPMG, com tempo de eco de 0,2 ms, 600 ecos, tempo de reciclagem de 1 segundo, e adquirir 16 espectros por amostra. Em seguida, ajustar a fase da componente real do espectro, que será usada nas análises multivariadas.

6) Curva de calibração por regressão dos mínimos quadrados parciais (partial least squares - PLS): para a determinação da qualidade do óleo em termos de índice de iodo (II), número de cetano $(\mathrm{CN})$ e viscosidade $(\mu)$, é necessário construir uma curva de calibração entre os valores determinados a partir dos dados cromatográficos, das equações 12.13 a 12.15 e os respectivos dados de RMN (600 dados do decaimento CPMG). Para obter a curva de calibração, utilizar um programa de análise multivariada, com PLS. Os dados de CPMG são agrupados em uma matriz de 600 variáveis (variável independente) de cada uma das amostras, enquanto os dados da concentração dos FAMEs são agrupados em uma matriz com a porcentagem de cada FAME para cada amostra (variável dependente). A performance da curva de calibração pode ser obtida realizando uma validação cruzada entre os dados obtido por cromatografia e os dados previstos por RMN. Com a curva de calibração para cada parâmetro de qualidade é possível prever esses mesmos parâmetros de qualidade do óleo em sementes intactas (amostras desconhecidas), a partir dos dados de RMN, obtidos com a sequência CPMG. 


\section{REFERÊNCIAS}

1. Levitt MH. Spin dynamics: basics of nuclear magnetic resonance. 2 ed. Chichester: John Wiley and Sons; 2009.

2. Abragam A. The principle of nuclear magnetism. Oxford: Clarendon Press; 1978.

3. Ernst RR, Bodenhausen G, Wokaun A. Principles of nuclear magnetic resonance in one and two dimensions. Oxford: Clarendon Press; 1990.

4. Farrar TC, Becker ED. Pulse and Fourier transform NMR: introduction to theory and methods. New York/London: Academic Press; 1971.

5. Colnago LA, Andrade FD, Souza AA, Azeredo RBdV, Lima AA, Cerioni LM, et al. Why is inline NMR rarely used as sensor in factory floor? Challenges and opportunities. Chem Eng Technol. 2014 Feb;37(2):191-203.

6. Gil VMS, Geraldes CFGC. Ressonância Magnética Nuclear: fundamentos, métodos e aplicações. Lisboa: Fundação Calouste Gulbenkian; 1987.

7. Nordon A, McGill CA, Littlejohn D. Process NMR spectrometry. Analyst. 2001;126(2):260-72.

8. Pedersen HT, Munck L, Engelsen SB. Low-field (1)H nuclear magnetic resonance and chemometrics combined for simultaneous determination of water, oil, and protein contents in oilseeds. Journal of the American Oil Chemists Society. 2000;77(10):1069-76. 9. Meiboom S, Gill D. Modified Spin-Echo Method for Measuring Nuclear Relaxation Times. Review of Scientific Instruments. 1958;29(8):688-91.

10. Carr HY, Purcell EM. Effects of Diffusion on Free Precession in Nuclear Magnetic Resonance Experiments. Physical Review. 1954;94(3):630-38.

11. Venancio T, Engelsberg M, Azeredo RBV, Alem NER, Colnago LA. Fast and simultaneous measurement of longitudinal and transverse NMR relaxation times in a single continuous wave free precession experiment. Journal of Magnetic Resonance. 2005;173(1):34-9.

12. Azeredo RBV. Espectroscopia de RMN CWFP [Tese de Doutorado]. São Carlos: Universidade de São Paulo; 2004.

13. Azeredo RBD, Engelsberg M, Colnago LA. Flow sensitivity and coherence in steadystate free spin precession. Physical Review. E 2001;64(1):16309-13.

14. de Andrade FD, Netto AM, Colnago LA. Use of Carr-Purcell pulse sequence with low refocusing flip angle to measure T-1 and T-2 in a single experiment. Journal of Magnetic Resonance. 2012;214:184-8.

15. Maus A, Hertlein C, Saalwächter K. A robust proton NMR method to investigate hard/soft ratios, crystallinity and component mobility in polymers. Macromol Chem Phys. 2006;207:1150-8.

16. Kimmich R, Fatkullin N. Polymer chain dynamics and NMR. Berlin/Heidelberg: Springer; 2004. 
17. Clauss J, Schmidt-Rohr K, Spiess HW. Determination of domain sizes in heterogeneous polymers by solid-state NMR. Acta Polym. 1993;44(1):1-7.

18. Saalwachter K. Proton multiple-quantum NMR for the study of chain dynamicsand structural constraints in polymeric soft materials. Prog Nucl Magn Reson Spectrosc. 2007;51:243-71.

19. Saalwachter K, Heuer A. Chain dynamics in elastomers asinvestigated by proton multiple-quantum NMR. Macromolecules. 2006;39:3291-303.

20. Saalwaechter K. Detection of heterogeneities in dry and swollen polymer networks by proton low-field NMR spectroscopy. Am Chem Soc. 2003;125:14684-5.

21. Goldman M, Shen L. Spin-Spin Relaxation in LaF3. Phys Rev. 1966;144:321-31.

22. Gaborieau M, Graf R, Spiess HW. Versatility of the dipolar filter selection: From $1 \mathrm{H}$ nuclear spin diffusion experiment to the measurement of nuclear Overhauser effect in homopolymer melts. Solid State Nucl Magn Reson. 2005;28:160-72.

23. Buda A, Demco DE, Bertmer B, Blumich B, Reining B, Keul H, et al. Domain sizes in heterogeneous polymers by spin diffusion using single-quantum and double-quantum dipolar filters. Solid State Nucl Magn Reson. 2003;24:39-67.

24. Duer M. Solid-State NMR Spectroscopy. 1st ed. Oxford: Blackwell; 2004.

25. Grohn H. A study of T1 Relaxation: From Relaxation Mechamisms to the Magnetic Resonance Imaging Contrast [Doctorate]. Joensuu: Kuopio University; 2003.

26. Hürlimann MD, Burcaw L, Song Y-Q. Quantitative characterization of food products by two-dimensional D-T2 and T1-T2 distribution functions in a static gradient. J. Colloid Interf Sci. 2006;297(1):303-11.

27. Song Y-Q, Venkataramanan L, Hürlimann MD, Flaum M, Frulla P, Straley C. T1-T2 Correlation Spectra Obtained Using a Fast Two-Dimensional Laplace Inversion. J Magn Res. 2002;154(2):261-8.

28. Bonastre A, Ors R, Capella JV, Fabra MJ, Peris M. In-line chemical analysis of wastewater: Present and future trends. Trac-Trends in Analytical Chemistry 2005;24(2):128-37.

29. Fox RW, Macdonald AT. Introdução à mecânica dos fluidos. 3 ed. Rio de Janeiro: Guanabara S. A.; 1985.

30. Suryan G. Nuclear resonance in flowing liquids. Proceedings of the Indian Academy of Sciences - Section A. 1951;33(2):107-11.

31. Singer JR. Blood Flow Rates by Nuclear Magnetic Resonance Measurements. Science. $1959 ; 130(3389): 1652-3$.

32. Ladner WR, Stacey AE. Measurement of Moisture in a Moving Coal Feed. British Journal of Applied Physics. 1962;13(3):136.

33. McIvor MC. A Flow Probe for Nuclear Magnetic Resonance Spectroscopy. Journal of Physics E-Scientific Instruments. 1969;2(3):292-3. 
34. Grover T, Singer JR. Nmr Spin-Echo Flow Measurements. Journal of Applied Physics. 1971;42(3):938-40.

35. Watanabe N, Niki E. Direct-Coupling of Ft-Nmr to High-Performance LiquidChromatography. Proceedings of the Japan Academy Series B-Physical and Biological Sciences. 1978;54(4):194-9.

36. Tellier C, Mariette F. On-line Applications in Food Science. Annual Reports on NMR Spectroscopy. In: G.A. Webb PSBaMJM, editor. V. 31. New York/London: Academic Press; 1995. p. 105-22.

37. Pearson RM, Ream LR, Job C, Adams J. The Use of Small Nuclear-MagneticResonance Spectrometers for Online Process-Control. Cereal Foods World. 1987;32(11):822-6.

38. Renou JP, Briguet A, Gatellier P, Kopp J. Determination of Fat and Water Ratios in Meat-Products by High-Resolution Nmr at 19.6 Mhz. International Journal of Food Science and Technology. 1987;22(2):169-72.

39. Nicholls CI, Santos AD. Hydrogen Transient Nuclear-Magnetic-Resonance for Industrial Moisture Sensing. Drying Technology. 1991;9(4):849-73.

40. Chen P, McCarthy MJ, Kauten R, Sarig Y, Han S. Maturity Evaluation of Avocados by Nmr Methods. Journal of Agricultural Engineering Research. 1993;55(3):177-87.

41. Zion B, Kim SM, McCarthy MJ, Chen P. Detection of pits in olives under motion by nuclear magnetic resonance. Journal of the Science of Food and Agriculture. 1997;75(4):496-502.

42. Hills BP, Wright KM. Motional relativity and industrial NMR sensors. Journal of Magnetic Resonance. 2006;178(2):193-205.

43. Hills BP, Clark CJ. Quality Assessment of Horticultural Products by NMR. Annual Reports on NMR Spectroscopy. V. 50. New York/London: Academic Press; 2003. p. 75-120.

44. Tu SS, Choi YJ, McCarthy MJ, McCarthy KL. Tomato quality evaluation by peak force and NMR spin-spin relaxation time. Postharvest Biology and Technology. 2007;44(2):157-64.

45. Hernández-Sánchez N, Hills BP, Barreiro P, Marigheto N. An NMR study on internal browning in pears. Postharvest Biology and Technology. 2007;44(3):260-70.

46. Colnago LA, Engelsberg M, Souza AA, Barbosa LL. High-throughput, non-destructive determination of oil content in intact seeds by continuous wave-free precession NMR. Analytical Chemistry. 2007;79(3):1271-4.

47. Correa CC, Forato LA, Colnago LA. High-throughput non-destructive nuclear magnetic resonance method to measure intramuscular fat content in beef. Analytical and Bioanalytical Chemistry. 2009;393(4):1357-60. 
48. Prestes RA, Colnago LA, Forato LA, Vizzotto L, Novotny EH, Carrilho E. A rapid and automated low resolution NMR method to analyze oil quality in intact oilseeds. Analytica Chimica Acta. 2007;596(2):325-9.

49. de Andrade FD, Netto AM, Colnago LA. Qualitative analysis by online nuclear magnetic resonance using Carr-Purcell-Meiboom-Gill sequence with low refocusing flip angles. Talanta. 2011;84(1):84-8. 
